\title{
Stanley decompositions and partitionable simplicial complexes
}

\author{
Jürgen Herzog • Ali Soleyman Jahan • \\ Siamak Yassemi
}

Received: 2 January 2007 / Accepted: 1 May 2007 /

Published online: 13 June 2007

(C) Springer Science+Business Media, LLC 2007

\begin{abstract}
We study Stanley decompositions and show that Stanley's conjecture on Stanley decompositions implies his conjecture on partitionable Cohen-Macaulay simplicial complexes. We also prove these conjectures for all Cohen-Macaulay monomial ideals of codimension 2 and all Gorenstein monomial ideals of codimension 3 .
\end{abstract}

Keywords Stanley decompositions · Partitionable simplicial complexes · Pretty clean modules

\section{Introduction}

In this paper we discuss the conjecture of Stanley [19] concerning a combinatorial upper bound for the depth of a $\mathbb{Z}^{n}$-graded module. Here we consider his conjecture only for $S / I$, where $I$ is a monomial ideal.

Let $K$ be a field, $S=K\left[x_{1}, \ldots, x_{n}\right]$ the polynomial ring in $n$ variables. Let $u \in S$ be a monomial and $Z$ a subset of $\left\{x_{1}, \ldots, x_{n}\right\}$. We denote by $u K[Z]$ the $K$-subspace

Dedicated to Takayuki Hibi on the occasion of his fiftieth birthday.

J. Herzog $(\bowtie) \cdot$ A.S. Jahan

Fachbereich Mathematik und Informatik, Universität Duisburg-Essen, Campus Essen, 45117

Essen, Germany

e-mail: juergen.herzog@uni-essen.de

A.S. Jahan

e-mail: ali.soleyman-jahan@stud.uni-duisburg-essen.de

S. Yassemi

Department of Mathematics, University of Tehran, P.O. Box 13145448, Tehran, Iran

S. Yassemi

Institute for Theoretical Physics and Mathematics (IPM), Tehran, Iran

e-mail: yassemi@ipm.ir 
of $S$ whose basis consists of all monomials $u v$, where $v$ is a monomial in $K[Z]$. The $K$-subspace $u K[Z] \subset S$ is called a Stanley space of dimension $|Z|$.

Let $I \subset S$ be a monomial ideal, and denote by $I^{c} \subset S$ the $K$-linear subspace of $S$ spanned by all monomials which do not belong to $I$. Then $S=I^{c} \oplus I$ as a $K$-vector space, and the residues of the monomials in $I^{c}$ form a $K$-basis of $S / I$.

A decomposition $\mathcal{D}$ of $I^{c}$ as a finite direct sum of Stanley spaces is called a Stanley decomposition of $S / I$. The minimal dimension of a Stanley space in the decomposition $\mathcal{D}$ is called the Stanley depth of $\mathcal{D}$, denoted $\operatorname{sdepth}(\mathcal{D})$.

We set $\operatorname{sdepth}(S / I)=\max \{\operatorname{sdepth}(\mathcal{D}): \mathcal{D}$ is a Stanley decomposition of $S / I\}$ and call this number the Stanley depth of $S / I$.

In [17, Conjecture 5.1] Stanley conjectured the inequality $\operatorname{sdepth}(S / I) \geq$ $\operatorname{depth}(S / I)$. We say that $I$ is a Stanley ideal if Stanley's conjecture holds for $S / I$.

Not many classes of Stanley ideals are known. Apel [3, Corollary 3] showed that all monomial ideals $I$ with $\operatorname{dim} S / I \leq 1$ are Stanley ideals. He also showed [3, Theorem 3 and Theorem 5] that all generic monomial ideals and all cogeneric CohenMacaulay monomial ideals are Stanley ideals, and Soleyman Jahan [15, Proposition 2.1] proved that all monomial ideals in a polynomial ring in $n$ variables of dimension less than or equal to 1 are Stanley ideals. The above facts imply in particular a result of Apel which says that all monomial ideals in the polynomial ring in three variables are Stanley ideals. The same result for four variables has been recently obtained in [2]. Moreover, Stanley's conjecture for small dimensions is also discussed in [1].

In [13] the authors attach to each monomial ideal a multi-complex and introduce the concept of shellable multi-complexes. In case $I$ is a squarefree monomial ideal, this concept of shellability coincides with the nonpure shellability introduced by Björner and Wachs [4]. It is shown in [13, Theorem 10.5] that if $I$ is pretty clean (see the definition in Sect. 3), then the multi-complex attached to $I$ is shellable and $I$ is a Stanley ideal. The concept of pretty clean modules is a generalization of clean modules introduced by Dress [8]. He showed that a simplicial complex is shellable if and only if its Stanley-Reisner ideal is clean.

We use these results to prove that any Cohen-Macaulay monomial ideal of codimension 2 and any Gorenstein monomial ideal of codimension 3 is a Stanley ideal, see Proposition 2.4 and Theorem 3.1. For the proof of Proposition 2.4, we observe that the polarization of a perfect codimension 2 ideal is shellable and show this by using Alexander duality and the result of [11] that any monomial ideal with 2-linear resolution has linear quotients. The proof of Theorem 3.1 is based on the structure theorem for Gorenstein monomial ideals given in [5]. It also uses the result, proved in Proposition 3.3, that a pretty clean monomial ideal remains pretty clean after applying a substitution replacing the variables by a regular sequence of monomials.

In the last section of this paper we introduce squarefree Stanley spaces and show in Proposition 4.2 that for a squarefree monomial ideal $I$, the Stanley decompositions of $S / I$ into squarefree Stanley spaces correspond bijectively to partitions into intervals of the simplicial complex whose Stanley-Reisner ideal is the ideal I. Stanley calls a simplicial complex $\Delta$ partitionable if there exists a partition $\Delta=\bigcup_{i=1}^{r}\left[F_{i}, G_{i}\right]$ of $\Delta$ such that for all intervals $\left[F_{i}, G_{i}\right]=\left\{F \in \Delta: F_{i} \subset F \subset G_{i}\right\}$ one has that $G_{i}$ is a facet of $\Delta$. We show in Corollary 4.5 that the Stanley-Reisner ideal $I_{\Delta}$ of a CohenMacaulay simplicial complex $\Delta$ is a Stanley ideal if and only if $\Delta$ is partitionable. In 
other words, Stanley's conjecture on Stanley decompositions implies his conjecture on partitionable simplicial complexes.

\section{Stanley decompositions}

Let $S=K\left[x_{1}, \ldots, x_{n}\right]$ be a polynomial ring and $I \subset S$ a monomial ideal. Note that $I$ and $I^{c}$ as well as all Stanley spaces are $K$-linear subspaces of $S$ with a basis that is a subset of monomials of $S$. For any $K$-linear subspace $U \subset S$ that is generated by monomials, we denote by $\operatorname{Mon}(U)$ the set of elements in the monomial basis of $U$. It is then clear that if $u_{i} K\left[Z_{i}\right], i=1, \ldots, r$, are Stanley spaces, then $I^{c}=$ $\bigoplus_{i=1}^{r} u_{i} K\left[Z_{i}\right]$ if and only if $\operatorname{Mon}\left(I^{c}\right)$ is the disjoint union of the sets $\operatorname{Mon}\left(u_{i} K\left[Z_{i}\right]\right)$.

Usually one has infinitely many different Stanley decompositions of $S / I$. For example, if $S=K\left[x_{1}, x_{2}\right]$ and $I=\left(x_{1} x_{2}\right)$, then for each integer $k \geq 1$ one has the Stanley decomposition

$$
\mathcal{D}_{k}: S / I=K\left[x_{2}\right] \oplus \bigoplus_{j=1}^{k} x_{1}^{j} K \oplus x_{1}^{k+1} K\left[x_{1}\right]
$$

of $S / I$. Each of these Stanley decompositions of $S / I$ has Stanley depth 0 , while the Stanley decomposition $K\left[x_{2}\right] \oplus x_{1} K\left[x_{1}\right]$ of $S / I$ has Stanley depth 1 .

Even though $S / I$ may have infinitely many different Stanley decompositions, all these decompositions have one property in common, as noted in [15, Sect. 2]. Indeed, if $\mathcal{D}$ is a Stanley decomposition of $S / I$ with $s=\operatorname{dim} S / I$, then the number of Stanley sets of dimension $s$ in $\mathcal{D}$ is equal to the multiplicity $e(S / I)$ of $S / I$.

There is also an upper bound for $\operatorname{sdepth}(S / I)$ known, namely

$$
\operatorname{sdepth}(S / I) \leq \min \{\operatorname{dim} S / P: P \in \operatorname{Ass}(S / I)\},
$$

see $[3$, Sect. 3]. Note that for $\operatorname{depth}(S / I)$ the same upper bound is valid. As a consequence of these observations, we have the following:

Corollary 2.1 Let I $\subset S$ be a monomial ideal such that $S / I$ is Cohen-Macaulay. Then the following conditions are equivalent:

(a) I is a Stanley ideal.

(b) There exists a Stanley decomposition $\mathcal{D}$ of $S / I$ such that each Stanley space in $\mathcal{D}$ has dimension $d=\operatorname{dim} S / I$.

(c) There exists a Stanley decomposition $\mathcal{D}$ of $S / I$ that has e(S/I) summands.

We now recall the notion of clean and pretty clean filtrations which will be used in the sequel. Let $I \subset S$ be a monomial ideal. According to [13], $S / I$ is called pretty clean if there exists a chain of monomial ideals such that:

(a) For all $j$, one has $I_{j} / I_{j-1} \cong S / P_{j}$, where $P_{j}$ is a monomial prime ideal.

(b) For all $i<j$ such that $P_{i} \subset P_{j}$, it follows that $P_{i}=P_{j}$. 
Dress [8] calls the ring $S / I$ clean if there exists a chain of ideals as above such that all the $P_{i}$ are minimal prime ideals of $I$. By an abuse of notation we call $I$ (pretty) clean if $S / I$ is (pretty) clean. Obviously, any clean ideal is pretty clean. In [13, Theorem 6.5] it is shown that if $I$ is pretty clean, then $I$ is a Stanley ideal, while Dress showed [8, Sect. 4] that if $I=I_{\Delta}$ for some simplicial complex $\Delta$, then $\Delta$ is shellable if and only if $I_{\Delta}$ is clean. In particular, it follows that $I_{\Delta}$ is a Stanley ideal if $\Delta$ is shellable.

The following result will be needed later in Sect. 3 .

Proposition 2.2 Let I $\subset S$ be a monomial complete intersection ideal. Then $S / I$ is clean. In particular, I is a Stanley ideal.

Proof Let $u \in S$ be a monomial. We call $\operatorname{supp}(u)=\left\{x_{i}: x_{i}\right.$ divides $\left.u\right\}$ the support of $u$. Now let $G(I)=\left\{u_{1}, \ldots, u_{m}\right\}$ be the unique minimal set of monomial generators of $I$. By our assumption, $u_{1}, \ldots, u_{m}$ is a regular sequence. This implies that $\operatorname{supp}\left(u_{i}\right) \cap \operatorname{supp}\left(u_{j}\right)=\emptyset$ for all $i \neq j$.

From the definition of the polarization of a monomial ideal (see, for example, [15]) it follows that for the polarized ideal $I^{p}=\left(u_{1}^{p}, \ldots, u_{m}^{p}\right)$ one again has $\operatorname{supp}\left(u_{i}^{p}\right) \cap$ $\operatorname{supp}\left(u_{j}^{p}\right)=\emptyset$ for all $i \neq j$.

Thus $J=I^{p}$ is a squarefree monomial ideal generated by the regular sequence of monomials $v_{1}, \ldots, v_{m}$ with $v_{i}=u_{i}^{p}$ for all $i$.

Let $\Delta$ be the simplicial complex whose Stanley-Reisner ideal $I_{\Delta}$ is equal to $J$. The Alexander dual $\Delta^{\vee}$ of $\Delta$ is defined to be the simplicial complex whose faces are $\{[n] \backslash F: F \notin \Delta\}$. The Stanley-Reisner ideal of $\Delta^{\vee}$ is minimally generated by all monomials $x_{i_{1}} \cdots x_{i_{k}}$, where $\left(x_{i_{1}}, \ldots, x_{i_{k}}\right)$ is a minimal prime ideal of $I_{\Delta}$.

In our case it follows that $I_{\Delta} \vee$ is minimally generated by the monomials of the form $x_{i_{1}} \ldots x_{i_{m}}$, where $x_{i_{j}} \in \operatorname{supp}\left(v_{j}\right)$ for $j=1, \ldots, m$. Thus we see that $I_{\Delta} \vee$ is the matroidal ideal of the transversal matroid attached to the sets $\operatorname{supp}\left(v_{1}\right), \ldots, \operatorname{supp}\left(v_{m}\right)$, see [7, Sect. 5]. In [14, Lemma 1.3] and [7, Section 5] it is shown that any polymatroidal ideal has linear quotients, and this implies that $\Delta$ is a shellable simplicial complex, see, for example, [12, Theorem 1.4]. Hence by the theorem of Dress quoted in the next section, $S / I_{\Delta}$ is clean. Now we use the result in [15, Theorem 3.10] which says that a monomial ideal is pretty clean (see the definition in Sect. 2) if and only if its polarization is clean. Therefore we conclude that $S / I$ is pretty clean. Since all prime ideals in a pretty clean filtration of $S / I$ are associated prime ideals of $S / I$ (see [13, Corollary 3.4]) and since $S / I$ is Cohen-Macaulay, the prime ideals in the filtration are minimal. Hence $S / I$ is clean. Thus from [13, Theorem 6.5] we conclude that $I$ is Stanley ideal.

Corollary 2.3 Let $I \subset S$ be a monomial ideal with depth $S / I \geq n-1$. Then $I$ is a Stanley ideal.

Proof The assumption implies that $I$ is a principal ideal. Thus the assertion follows from Proposition 2.2.

With the same techniques as in the proof of Proposition 2.2 we can show the following: 
Proposition 2.4 Let I $\subset S$ be a monomial ideal that is perfect and of codimension 2. Then $S / I$ is clean. In particular, I is a Stanley ideal.

Proof We will show that the polarized ideal $I^{p}$ defines a shellable simplicial complex. Then, as in the proof of Proposition 2.2, it follows that $S / I$ is clean. Note that $I^{p}$ is a perfect squarefree monomial ideal of codimension 2 . Let $\Delta$ be the simplicial complex defined by $I^{p}$. By the Eagon-Reiner theorem [9] and a result of Terai [20], the ideal $I_{\Delta} \vee$ has a 2-linear resolution. Now we use the fact, proved in [11, Theorem 3.2], that an ideal with a 2-linear resolution has linear quotients, which in turn implies that $\Delta$ is shellable, as desired.

Combining the preceding results with Apel's result according to which all monomial ideals with $\operatorname{dim} S / I \leq 1$ are Stanley ideals, we obtain the following:

Corollary 2.5 Let I $\subset S$ be a monomial ideal. If $n \leq 4$ and $S / I$ is Cohen-Macaulay, then I is a Stanley ideal.

\section{Gorenstein monomial ideals of codimension 3}

As the main result of this section, we will show the following:

Theorem 3.1 Each Gorenstein monomial ideal of codimension 3 is a Stanley ideal.

The proof of this result is based on the following structure theorem that can be found in [5].

Theorem 3.2 Let $I \subset S$ be a monomial Gorenstein ideal of codimension 3. Then $|G(I)|$ is an odd number, say $|G(I)|=2 m+1$, and there exists a regular sequence of monomials $u_{1}, \ldots, u_{2 m+1}$ in $S$ such that

$$
G(I)=\left\{u_{i} u_{i+1} \cdots u_{i+m-1}: i=1, \ldots, 2 m+1\right\},
$$

where $u_{i}=u_{i-2 m-1}$ whenever $i>2 m+1$.

We now show

Proposition 3.3 Let $I \subset T=K\left[y_{1}, \ldots, y_{r}\right]$ be a monomial ideal such that $T / I$ is (pretty) clean. Let $u_{1}, \ldots, u_{r} \in S=K\left[x_{1}, \ldots, x_{n}\right]$ be a regular sequence of monomials, and let $\varphi: T \rightarrow S$ be the K-algebra homomorphism with $\varphi\left(y_{j}\right)=u_{j}$ for $j=1, \ldots, r$. Then $S / \varphi(I) S$ is (pretty) clean.

Proof Let $I=I_{0} \subset I_{1} \subset \cdots \subset I_{m}=T$ be a pretty clean filtration $\mathcal{F}$ of $T / I$ with $I_{k} / I_{k-1}=T / P_{k}$ for all $k$.

Observe that the $K$-algebra homomorphism $\varphi: T \rightarrow S$ is flat, since $u_{1}, \ldots, u_{r}$ is a regular sequence. Hence if we set $J_{k}=\phi\left(I_{k}\right) S$ for $k=1, \ldots, m$, then we obtain the filtration $\varphi(I) S=J_{0} \subset J_{1} \subset \cdots \subset J_{m}=S$ with $J_{k} / J_{k-1} \cong S / \varphi\left(P_{k}\right) S$. 
Suppose that $P_{k}=\left(y_{i_{1}}, \ldots, y_{i_{k}}\right)$; then $\varphi\left(P_{k}\right) S=\left(u_{i_{1}}, \ldots, u_{i_{k}}\right)$. In other words, $\varphi\left(P_{k}\right) S$ is a monomial complete intersection, and hence by Proposition 2.2 we have that $S / \varphi\left(P_{k}\right) S$ is clean. Therefore there exists a prime filtration $J_{k}=J_{k_{0}} \subset J_{k_{1}} \subset$ $\cdots \subset J_{k_{r_{k}}}=J_{k+1}$ such that $J_{k_{i}} / J_{k_{i-1}} \cong S / P_{k_{i}}$, where $P_{k_{i}}$ is a minimal prime ideal of $\varphi\left(P_{k}\right) S$. Since $\varphi\left(P_{k}\right) S=\left(u_{i_{1}}, \ldots, u_{i_{t_{k}}}\right) S$ is a complete intersection, all minimal prime ideals of $\varphi\left(P_{k}\right)$ have height $t_{k}$.

Composing the prime filtrations of $J_{k} / J_{k-1}$, we obtain a prime filtration of $S / \varphi(I) S$. We claim that this prime filtration is (pretty) clean. In fact, let $P_{k_{i}}$ and $P_{\ell_{j}}$ be two prime ideals in the support of this filtration. We have to show that if $P_{k_{i}} \subset P_{\ell_{j}}$ for $k<\ell$ or $P_{k_{i}} \subset P_{\ell_{j}}$ for $k=\ell$ and $i<j$, then $P_{k_{i}}=P_{\ell_{j}}$. In the case $k=\ell$, we have height $\left(P_{k_{i}}\right)=\operatorname{height}\left(P_{\ell_{j}}\right)=t_{k}$, and the assertion follows. In the case $k<\ell$, by using the fact that $\mathcal{F}$ is a pretty clean filtration, we have that $P_{k}=P_{\ell}$ or $P_{k} \not \subset P_{\ell}$. In the first case, the prime ideals $P_{k_{i}}$ and $P_{\ell_{j}}$ have the same height, and the assertion follows. In the second case, there exists a variable $y_{g} \in P_{k} \backslash P_{\ell}$. Then the monomial $u_{g}$ belongs to $\varphi\left(P_{k}\right) S$ but not to $\varphi\left(P_{\ell}\right) S$. This implies that $P_{k_{i}}$ contains a variable which belongs to the support of $u_{g}$. However this variable cannot be a generator of $P_{\ell_{j}}$, because the support of $u_{g}$ is disjoint from the support of all the monomial generators of $\varphi\left(P_{\ell}\right) S$. This shows that $P_{k_{i}} \not \subset P_{\ell_{j}}$.

Corollary 3.4 Let $\Delta$ be a shellable simplicial complex and $I_{\Delta} \subset T=K\left[y_{1}, \ldots, y_{r}\right]$ its Stanley-Reisner ideal. Furthermore, let $u_{1}, \ldots, u_{r} \subset S=K\left[x_{1}, \ldots, x_{n}\right]$ be a regular sequence of monomials, and let $\varphi\left(y_{i}\right)=u_{i}$ for $i=1, \ldots, r$. Then $\varphi\left(I_{\Delta}\right) S$ is a Stanley ideal.

Proof By the theorem of Dress, the ring $T / I_{\Delta}$ is clean. Therefore, $S / \varphi\left(I_{\Delta}\right) S$ is again clean by Proposition 3.3. In particular, $S / \varphi\left(I_{\Delta}\right) S$ is pretty clean, which according to [13, Theorem 6.5] implies that $\varphi\left(I_{\Delta}\right) S$ is a Stanley ideal.

Proof of Theorem 3.1 Let $\Delta$ be the simplicial complex whose Stanley-Reisner ideal

$$
I_{\Delta} \subset T=K\left[y_{1}, \ldots, y_{2 m+1}\right]
$$

is generated by the monomials $y_{i} y_{i+1} \cdots y_{i+m-1}, i=1, \ldots, 2 m+1$, where $y_{i}=$ $y_{i-2 m-1}$ whenever $i>2 m+1$, and let $u_{1}, \ldots, u_{2 m+1} \subset S=K\left[x_{1}, \ldots, x_{n}\right]$ be the regular sequence given in Theorem 3.1. Then we have $I=\varphi\left(I_{\Delta}\right) S$ where $\varphi\left(y_{j}\right)=u_{j}$ for all $j$. Therefore, by Corollary 3.4, it suffices to show that $\Delta$ is shellable.

Identifying the vertex set of $\Delta$ with $[2 m+1]=\{1, \ldots, 2 m+1\}$ and observing that $I_{\Delta}$ is of codimension 3 , it is easy to see that $F \subset[2 m+1]$ is a facet of $\Delta$ if and only if $F=[2 m+1] \backslash\left\{a_{1}, a_{2}, a_{3}\right\}$ with

$$
a_{2}-a_{1}<m+1, \quad a_{3}-a_{2}<m+1, \quad a_{3}-a_{1}>m .
$$

We denote the facet $[2 m+1] \backslash\left\{a_{1}, a_{2}, a_{3}\right\}$ by $\mathrm{F}\left(a_{1}, a_{2}, a_{3}\right)$.

We will show that $\Delta$ is shellable with respect to the lexicographic order. Note that $\mathrm{F}\left(a_{1}, a_{2}, a_{3}\right)<\mathrm{F}\left(b_{1}, b_{2}, b_{3}\right)$ in the lexicographic order if and only if either $b_{1}<a_{1}$, or $b_{1}=a_{1}$ and $b_{2}<a_{2}$, or $a_{1}=b_{1}, a_{2}=b_{2}$, and $b_{3}<a_{3}$.

In order to prove that $\Delta$ is shellable we have to show that if $F=\mathrm{F}\left(a_{1}, a_{2}, a_{3}\right)$ and $G=\mathrm{F}\left(b_{1}, b_{2}, b_{3}\right)$ with $F<G$, then there exists $c \in G \backslash F$ and some facet $H$ such that $H<G$ and $G \backslash H=\{c\}$. 
We know that $|G \backslash F| \leq 3$. If $|G \backslash F|=1$, then there is nothing to prove. In the following we discuss the cases $|G \backslash F|=2$ and $|G \backslash F|=3$. The discussion of these cases is somewhat tedious but elementary. For the convenience of the reader, we list all the possible cases.

Case $1:|G \backslash F|=2$.

(i) If $b_{1}=a_{1}<b_{2}<a_{2}$, then we choose $H=\left(G \backslash\left\{a_{2}\right\}\right) \cup\left\{b_{2}\right\}$.

(ii) If $b_{1}<b_{2}=a_{1}$ or $b_{1}<b_{2}<a_{1}<a_{2}=b_{3}<a_{3}$, then we choose $H=(G \backslash$ $\left.\left\{a_{3}\right\}\right) \cup\left\{b_{1}\right\}$.

(iii) If $b_{1}<a_{1}<b_{2}<a_{2}=b_{3}<a_{3}$, we consider the following two subcases:

For $a_{3}-b_{2}<m+1$, we choose $H=\left(G \backslash\left\{a_{3}\right\}\right) \cup\left\{b_{3}\right\}$.

For $a_{3}-b_{2} \geq m+1$, we choose $H=\left(G \backslash\left\{a_{3}\right\}\right) \cup\left\{b_{1}\right\}$.

(iv) If $b_{1}<a_{1}<a_{2}=b_{2}<b_{3}<a_{3}$, then we choose $H=\left(G \backslash\left\{a_{3}\right\}\right) \cup\left\{b_{3}\right\}$.

(v) If $b_{1}<a_{1}<a_{2}=b_{2}<a_{3}<b_{3}$ or $b_{1}<a_{1}<a_{2}<a_{3}=b_{2}<b_{3}$, then we choose $H=\left(G \backslash\left\{a_{1}\right\}\right) \cup\left\{b_{1}\right\}$.

Case 2: $|G \backslash F|=3$.

(i) If $b_{1}<a_{1}<a_{2}<a_{3}<b_{3}$, then we choose $H=\left(G \backslash\left\{a_{1}\right\}\right) \cup\left\{b_{1}\right\}$.

(ii) If $b_{1}<b_{2}<b_{3}<a_{1}<a_{2}<a_{3}$ or $b_{1}<b_{2}<a_{1}<a_{2}<a_{3}$ and $a_{1}<b_{3}$, then we choose $H=\left(G \backslash\left\{a_{1}\right\}\right) \cup\left\{b_{2}\right\}$.

(iii) If $b_{1}<a_{1}<b_{2}<b_{3}<a_{2}<a_{3}$, then we choose $H=\left(G \backslash\left\{a_{2}\right\}\right) \cup\left\{b_{3}\right\}$.

(iv) If $b_{1}<a_{1}<b_{2}<a_{2}<b_{3}<a_{3}$, we consider the following two subcases:

For $a_{3}-b_{2}<m+1$, we choose $H=\left(G \backslash\left\{a_{3}\right\}\right) \cup\left\{b_{3}\right\}$.

For $a_{3}-b_{2} \geq m+1$, we choose $H=\left(G \backslash\left\{a_{3}\right\}\right) \cup\left\{b_{1}\right\}$.

(v) If $b_{1}<a_{1}<a_{2}<b_{2}<b_{3}<a_{3}$, then we choose $H=\left(G \backslash\left\{a_{3}\right\}\right) \cup\left\{b_{3}\right\}$.

Combining the result of Theorem 3.1 with Corollary 2.3, Proposition 2.4, and the result of Apel [3, Corollary 3], we obtain:

Corollary 3.5 Let I $\subset S$ be monomial ideal. If $n \leq 5$ and $S / I$ is Gorenstein, then I is a Stanley ideal.

\section{Squarefree Stanley decompositions and partitions of simplicial complexes}

A Stanley space $u K[Z]$ is called a squarefree Stanley space if $u$ is a squarefree monomial and $\operatorname{supp}(u) \subseteq Z$. We shall use the following notation: for $F \subseteq[n]$, we set $x_{F}=\prod_{i \in F} x_{i}$ and $Z_{F}=\left\{x_{i}: i \in F\right\}$. Then a Stanley space is squarefree if and only if it is of the form $x_{F} K\left[Z_{G}\right]$ with $F \subseteq G \subseteq[n]$.

A Stanley decomposition of $S / I$ is called a squarefree Stanley decomposition of $S / I$ if all Stanley spaces in the decomposition are squarefree.

Lemma 4.1 Let I $\subset S$ be a monomial ideal. The following conditions are equivalent:

(a) I is a squarefree monomial ideal.

(b) S/I has a squarefree Stanley decomposition. 
Proof (a) $\Longrightarrow$ (b) We may view $I$ as the Stanley-Reisner ideal of some simplicial complex $\Delta$. With each $F \in \Delta$ we associate the squarefree Stanley space $x_{F} K\left[Z_{F}\right]$. We claim that $\bigoplus_{F \in \Delta} x_{F} K\left[Z_{F}\right]$ is a (squarefree) Stanley decomposition of $S / I$. Indeed, a monomial $u \in S$ belongs to $I^{c}$ if and only if $\operatorname{supp}(u) \in \Delta$, and these monomials form a $K$-basis for $I^{c}$. On the other hand, a monomial $u \in S$ belongs to $x_{F} K\left[Z_{F}\right]$ if and only if $\operatorname{supp}(u)=F$. This shows that $I^{c}=\bigoplus_{F \in \Delta} x_{F} K\left[Z_{F}\right]$.

(b) $\Longrightarrow$ (a) Let $\bigoplus_{i} u_{i} K\left[Z_{i}\right]$ be a squarefree Stanley decomposition of $S / I$. Assume that $I$ is not a squarefree monomial ideal. Then there exists $u \in G(I)$ that is not squarefree, and we may assume that $x_{1}^{2} \mid u$. Then $u^{\prime}=u / x_{1} \in I^{c}$, and hence there exists $i$ such that $u^{\prime} \in u_{i} K\left[Z_{i}\right]$. Since $x_{1} \mid u^{\prime}$, it follows that $x_{1} \in Z_{i}$. Therefore $u \in u_{i} K\left[Z_{i}\right] \subset I^{c}$, a contradiction.

Let $\Delta$ be a simplicial complex of dimension $d-1$ on the vertex set $V=$ $\left\{x_{1}, \ldots, x_{n}\right\}$. A subset $\mathcal{I} \subset \Delta$ is called an interval if there exist faces $F, G \in \Delta$ such that $\mathcal{I}=\{H \in \Delta: F \subseteq H \subseteq G\}$. We denote this interval given by $F$ and $G$ also by $[F, G]$ and call $\operatorname{dim} G-\operatorname{dim} F$ the rank of the interval. A partition $\mathcal{P}$ of $\Delta$ is a presentation of $\Delta$ as a disjoint union of intervals. The $r$-vector of $\mathcal{P}$ is the integer vector $r=\left(r_{0}, r_{1}, \ldots, r_{d}\right)$, where $r_{i}$ is the number of intervals of rank $i$.

Proposition 4.2 Let $\mathcal{P}$ : $\Delta=\bigcup_{i=1}^{r}\left[F_{i}, G_{i}\right]$ be a partition of $\Delta$. Then

(a) $D(\mathcal{P})=\bigoplus_{i=1}^{r} x_{F_{i}} K\left[Z_{G_{i}}\right]$ is a squarefree Stanley decomposition of $S / I$.

(b) The map $\mathcal{P} \mapsto D(\mathcal{P})$ establishes a bijection between partitions of $\Delta$ and squarefree Stanley decompositions of $S / I$.

Proof (a) Since each $x_{F_{i}} K\left[Z_{G_{i}}\right]$ is a squarefree Stanley space, it suffices to show that $I^{c}$ is indeed the direct sum of the Stanley spaces $x_{F_{i}} K\left[Z_{G_{i}}\right]$. Let $u \in \operatorname{Mon}\left(I^{c}\right)$; then $H=\operatorname{supp}(u) \in \Delta$. Since $\mathcal{P}$ is a partition of $\Delta$, it follows that $H \in\left[F_{i}, G_{i}\right]$ for some $i$. Therefore, $u=x_{F_{i}} u^{\prime}$ for some monomial $u^{\prime} \in K\left[Z_{G_{i}}\right]$. This implies that $u \in$ $x_{F_{i}} K\left[Z_{G_{i}}\right]$. This shows that $\operatorname{Mon}\left(I^{c}\right)$ is the union of sets $\operatorname{Mon}\left(x_{F_{i}} K\left[Z_{G_{i}}\right]\right)$. Suppose that there exists a monomial $u \in x_{F_{i}} K\left[Z_{G_{i}}\right] \cap x_{F_{j}} K\left[Z_{G_{j}}\right]$. Then $\operatorname{supp}(u) \in\left[F_{i}, G_{i}\right] \cap$ $\left[F_{j}, G_{j}\right]$. This is only possible if $i=j$, since $\mathcal{P}$ is partition of $\Delta$.

(b) Let $\left[F_{i}, G_{i}\right]$ and $\left[F_{j}, G_{j}\right]$ be two intervals. Then $x_{F_{i}} K\left[Z_{G_{i}}\right]=x_{F_{j}} K\left[Z_{G_{j}}\right]$ if and only if $\left[F_{i}, G_{i}\right]=\left[F_{j}, G_{j}\right]$. Indeed, if $x_{F_{i}} K\left[Z_{G_{i}}\right]=x_{F_{j}} K\left[Z_{G_{j}}\right]$, then $x_{F_{j}} \in$ $x_{F_{i}} K\left[Z_{G_{i}}\right]$, and hence $x_{F_{i}} \mid x_{F_{j}}$. By symmetry we also have $x_{F_{j}} \mid x_{F_{i}}$. In other words, $F_{i}=F_{j}$, and it also follows that $K\left[Z_{G_{i}}\right]=K\left[Z_{G_{j}}\right]$. This implies that $G_{i}=G_{j}$. These considerations show that $\mathcal{P} \mapsto D(\mathcal{P})$ is injective.

On the other hand, let $\mathcal{D}: S / I=\bigoplus_{i=1}^{r} x_{F_{i}} K\left[Z_{G_{i}}\right]$ be an arbitrary squarefree Stanley decomposition of $S / I$. By the definition of a squarefree Stanley set we have $F_{i} \subseteq G_{i}$, and since $x_{F_{i}} K\left[Z_{G_{i}}\right] \subset I^{c}$, it follows that $G_{i} \in \Delta$. Hence $\left[F_{i}, G_{i}\right]$ is an interval of $\Delta$, and a squarefree monomial $x_{F}$ belongs to $x_{F_{i}} K\left[Z_{G_{i}}\right]$ if and only if $F \in\left[F_{i}, G_{i}\right]$.

Let $F \subset \Delta$ be an arbitrary face. Then $x_{F} \in \operatorname{Mon}\left(I^{c}\right)=\bigcup_{i=1}^{r} \operatorname{Mon}\left(x_{F_{i}} K\left[Z_{G_{i}}\right]\right)$. Hence the squarefree monomial $x_{F}$ belongs to $x_{F_{i}} K\left[Z_{G_{i}}\right]$ for some $i$, and hence $F \in$ $\left[F_{i}, G_{i}\right]$. This shows that $\bigcup_{i=1}^{r}\left[F_{i}, G_{i}\right]=\Delta$. Suppose that $F \in\left[F_{i}, G_{i}\right] \cap\left[F_{j}, G_{j}\right]$. Then $x_{F} \in x_{F_{i}} K\left[Z_{G_{i}}\right] \cap x_{F_{j}} K\left[Z_{G_{j}}\right]$, a contradiction. Hence we see that $\mathcal{P}: \Delta=$ $\bigcup_{i=1}^{r}\left[F_{i}, G_{i}\right]$ is a partition of $\Delta$ with $D(\mathcal{P})=\mathcal{D}$. 
Now let $I \subset S$ be a squarefree monomial ideal. Then we set

$\operatorname{sqdepth}(S / I)=\max \{\operatorname{sdepth}(\mathcal{D}): \mathcal{D}$ is a squarefree Stanley decomposition of $S / I\}$

and call this number the squarefree Stanley depth of $S / I$.

As the main result of this section, we have the following:

Theorem 4.3 Let $I \subset S$ be a squarefree monomial ideal. Then $\operatorname{sqdepth}(S / I)=$ $\operatorname{sdepth}(S / I)$.

Proof Let $\mathcal{D}$ be any Stanley decomposition of $S / I$, and let $\Delta$ be the simplicial complex satisfying $I=I_{\Delta}$. For each $F \in \Delta$, we have $x_{F} \in I^{c}$. Hence there exists a summand $u K[Z]$ such that $x_{F} \in u K[Z]$. Since $x_{F}$ is squarefree, it follows that $u=x_{G}$ is squarefree and $F \subseteq G \cup Z$. Let $\mathcal{D}^{\prime}$ be the sum of those Stanley spaces $u K[Z]$ in $\mathcal{D}$ for which $u$ is a squarefree monomial. Then this sum is direct. Therefore the intervals $[G, G \cup Z]$ corresponding to the summands in $\mathcal{D}^{\prime}$ are pairwise disjoint. On the other hand, these intervals cover $\Delta$, as we have seen before, and hence form a partition of $\mathcal{P}$ of $\Delta$. From the construction of $\mathcal{P}$ it follows that sqdepth $D(\mathcal{P}) \geq \operatorname{sdepth} \mathcal{D}$. This $\operatorname{shows}$ that $\operatorname{sqdepth}(S / I) \geq \operatorname{sdepth}(S / I)$. The other inequality $\operatorname{sqdepth}(S / I) \leq \operatorname{sdepth}(S / I)$ is obvious.

Corollary 4.4 Let $\Delta$ be a simplicial complex. Then the following conditions are equivalent:

(a) $I_{\Delta}$ is a Stanley ideal.

(b) There exists a partition $\Delta=\bigcup_{i=1}^{r}\left[F_{i}, G_{i}\right]$ with $\left|G_{i}\right| \geq \operatorname{depth} K[\Delta]$ for all $i$.

Let $\Delta$ be a simplicial complex and $\mathcal{F}(\Delta)$ its set of facets. Stanley calls a simplicial complex $\Delta$ partitionable if there exists a partition $\Delta=\bigcup_{i=1}^{r}\left[F_{i}, G_{i}\right]$ with $\mathcal{F}(\Delta)=\left\{G_{1}, \ldots, G_{r}\right\}$. We call a partition with this property a nice partition. Stanley conjectures [18, Conjecture 2.7] (see also [19, Problem 6]) that each CohenMacaulay simplicial complex is partitionable. In view of Corollary 2.1, it follows that the conjecture on Stanley decompositions implies the conjecture on partitionable simplicial complexes. More precisely, we have the following:

Corollary 4.5 Let $\Delta$ be a Cohen-Macaulay simplicial complex with the h-vector $\left(h_{0}, h_{1}, \ldots, h_{d}\right)$. Then the following conditions are equivalent:

(a) $I_{\Delta}$ is a Stanley ideal.

(b) $\Delta$ is partitionable.

(c) $\Delta$ admits a partition whose $r$-vector satisfies $r_{i}=h_{d-i}$ for $i=0, \ldots, d$.

(d) $\Delta$ admits a partition into e $(K[\Delta])$ intervals.

Moreover, any nice partition of $\Delta$ satisfies conditions (c) and (d).

Proof (a) $\Longleftrightarrow$ (b) follows from Corollary 4.4. In order to prove the implication (b) $\Longrightarrow\left(\right.$ c), consider a nice partition $\Delta=\bigcup_{i=1}^{r}\left[F_{i}, G_{i}\right]$ of $\Delta$. From this decompo- 
sition, the $f$-vector of $\Delta$ can be computed by the formula

$$
\sum_{i=0}^{d} f_{i-1} t^{i}=\sum_{i=0}^{d} r_{i} t^{d-i}(1+t)^{i}
$$

On the other hand, one has

$$
\sum_{i=0}^{d} f_{i-1} t^{i}=\sum_{i=0}^{d} h_{i} t^{i}(1+t)^{d-i},
$$

see [6, p. 213]. Comparing the coefficients, the assertion follows.

The implication (c) $\Longrightarrow$ (d) follows from the fact that $e(K[\Delta])=\sum_{i=0}^{d} h_{i}$, see [6, Proposition 4.1.9]. Finally, (d) $\Longrightarrow$ (a) follows from Corollary 2.1.

We conclude this section with some explicit examples. Recall that constructibility, a generalization of shellability, is defined recursively as follows: (i) a simplex is constructible, (ii) if $\Delta_{1}$ and $\Delta_{2}$ are $d$-dimensional constructible complexes and their intersection is a $(d-1)$-dimensional constructible complex, then their union is constructible. In this definition, if in the recursion we restrict $\Delta_{2}$ always to be a simplex, then the definition becomes equivalent to that of (pure) shellability. The notion of constructibility for simplicial complexes appears in [16]. It is known and easy to see that

$$
\text { shellable } \Rightarrow \text { constructible } \Rightarrow \text { Cohen-Macaulay. }
$$

Since any shellable simplicial complex is partitionable (see [18, p. 79]), it is natural to ask whether any constructible complex is partitionable. This question is a special case of Stanley's conjecture that says that Cohen-Macaulay simplicial complexes are partitionable. We do not know the answer yet! In the following we present some examples where the complexes are not shellable or are not Cohen-Macaulay but the ideals related to these simplicial complexes are Stanley ideals.

Example 4.6 The following example of a simplicial complex is due to Masahiro Hachimori [10]. The simplicial complex $\Delta$ described by the next figure is 2-dimensional and nonshellable but constructible. It is constructible, because if we divide the simplicial complex by the bold line, we obtain two shellable complexes, and their intersection is a shellable 1-dimensional simplicial complex.

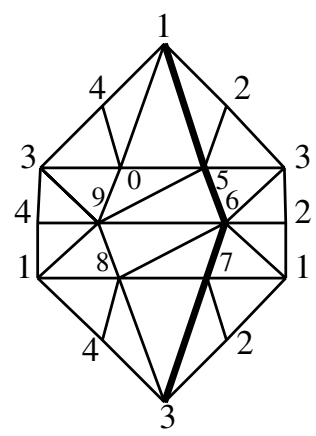


Indeed we can write $\Delta=\Delta_{1} \cup \Delta_{2}$, where the shelling order of the facets of $\Delta_{1}$ is given by

$148,149,140,150,189,348,349,378,340,390,590,569,689,678$

and that of $\Delta_{2}$ is given by

$125,126,127,167,235,236,237,356$.

We use the following principle to construct a partition of $\Delta$ : suppose that $\Delta_{1}$ and $\Delta_{2}$ are $d$-dimensional partitionable simplicial complexes and that $\Gamma=\Delta_{1} \cap \Delta_{2}$ is a $(d-1)$-dimensional pure simplicial complex. Let $\Delta_{1}=\bigcup_{i=1}^{r}\left[K_{i}, L_{i}\right]$ be a nice partition of $\Delta_{1}$, and $\Delta_{2}=\bigcup_{i=1}^{s}\left[F_{i}, G_{i}\right]$ a nice partition of $\Delta_{2}$. Suppose that, for each $i$, the set $\left[F_{i}, G_{i}\right] \backslash \Gamma$ has a unique minimal element $H_{i}$. Then $\Delta_{1} \cup \Delta_{2}=$ $\bigcup_{i=1}^{r}\left[K_{i}, L_{i}\right] \cup \bigcup_{i=1}^{s}\left[H_{i}, G_{i}\right]$ is a nice partition of $\Delta_{1} \cup \Delta_{2}$. Notice that $\left[F_{i}, G_{i}\right] \backslash \Gamma$ has a unique minimal element if and only if, for all $F \in\left[F_{i}, G_{i}\right] \cap \Gamma$, there exists a facet $G$ of $\Gamma$ with $F \subseteq G \subset G_{i}$.

Suppose that $\Delta_{2}$ is shellable with shelling $G_{1}, \ldots, G_{s}$. Let $F_{i}$ be the unique minimal subface of $G_{i}$ that is not a subface of any $G_{j}$ with $j<i$. Then $\Delta_{2}=$ $\bigcup_{i=1}^{s}\left[F_{i}, G_{i}\right]$ is the nice partition induced by this shelling. The above discussions then show that $\Delta_{1} \cup \Delta_{2}$ is partitionable if, for all $i$ and all $F \in \Gamma$ such that $F \subset G_{i}$ and $F \not \subset G_{j}$ for $j<i$, there exists a facet $G \in \Gamma$ with $F \subseteq G \subset G_{i}$.

In our particular case the shelling of $\Delta_{1}$ induces the following partition of $\Delta_{1}$ :

$$
\text { [Ø, 148], [9, 149], [0, 140], [5, 150], [89, 189], [3, 348], [39, 349], [7, 378], }
$$

$$
\text { [30, 340], [90, 390], [59, 590], [6, 569], [68, 689], [67, 678], }
$$

and the shelling of $\Delta_{2}$ induces the following partition of $\Delta_{2}$ :

$$
\text { [Ø, 125], [6, 126], [7, 127], [67, 167], [3, 235], [36, 236], [37, 237], [56, 356]. }
$$

The facets of $\Gamma=\Delta_{1} \cap \Delta_{2}$ are: $15,56,67,73$.

The restrictions of the intervals of this partition of $\Delta_{2}$ to the complement of $\Gamma$ do not all give intervals. For example, we have $[6,126] \backslash \Gamma=\{16,26,126\}$. This set has two minimal elements, and hence is not an interval. On the other hand, the partition of $\Delta_{2}$ (which is not induced from a shelling)

$$
\text { [Ø, 237], [1, 125], [5, 356], [6, 167], [17, 127], [25, 235], [26, 126], [36, 236] }
$$

restricted to the complement of $\Gamma$ yields the intervals

$$
[2,237],[12,125],[35,356],[16,167],[17,127],[25,235],[26,126],[36,236] \text {, }
$$

which together with the intervals of the partition of $\Delta_{1}$ give us a partition of $\Delta$.

Example 4.7 (The Dunce hat) The Dunce hat is the topological space obtained from the solid triangle $a b c$ by identifying the oriented edges $\overrightarrow{a b}, \overrightarrow{b c}$, and $\overrightarrow{a c}$. The following is a triangulation of the Dunce hat using 8 vertices. 


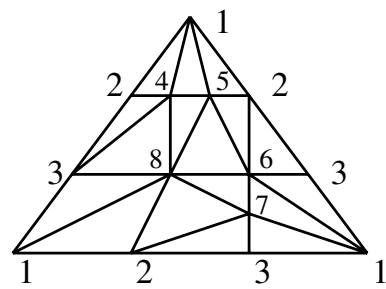

The facets arising from this triangulation are

124, 125, 145, 234, 348, 458, 568, 256, 236, 138, 128, 278, 678, 237, 137, 167, 136.

It is known that the simplicial complex corresponding to this triangulation is not shellable (not even constructible), but it is Cohen-Macaulay, see [10], and has the following partition:

$[\emptyset, 124],[3,234],[5,145],[6,236],[7,137],[8,348],[13,138],[16,136],[18,128]$,

[25, 125], [27, 237], [28, 278], [56, 256], [67, 167], [68, 568], [78, 678], [58, 458].

Therefore we again have $\operatorname{depth}(\Delta)=\operatorname{dim}(\Delta)=\operatorname{sdepth}(\Delta)=3$.

Example 4.8 (The Cylinder) The ideal $I=\left(x_{1} x_{4}, x_{2} x_{5}, x_{3} x_{6}, x_{1} x_{3} x_{5}, x_{2} x_{4} x_{6}\right) \subset$ $K\left[x_{1}, \ldots, x_{6}\right]$ is the Stanley-Reisner ideal of the triangulation of the cylinder shown in the next figure. The corresponding simplicial complex $\Delta$ is Buchsbaum but not Cohen-Macaulay.

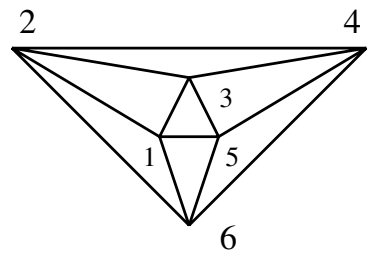

The facets of $\Delta$ are $123,126,156,234,345,456$, and it has the following partition:

$$
\text { [Ø, 123], [4, 234], [5, 345], [6, 456], [15, 156], [16, 126], [26, 26]. }
$$

Therefore we have $\operatorname{depth}(\Delta)=\operatorname{sdepth}(\Delta)=2<3=\operatorname{dim}(\Delta)$. Although $\Delta$ is not partitionable, $I_{\Delta}$ is a Stanley ideal.

Acknowledgements This paper was prepared during the third author's visit to the Universität DuisburgEssen, where he was on sabbatical leave from the University of Tehran. He would like to thank Deutscher Akademischer Austausch Dienst (DAAD) for the partial support. He also thanks the authorities of the Universität Duisburg-Essen for their hospitality during his stay there.

\section{References}

1. Ahmad, S., \& Popescu, D. (2007). Sequentially Cohen-Macaulay monomial ideals of embedding dimension four. math.AC/0702569. 
2. Anwar, I., \& Popescu, D. (2007). Stanley conjecture in small embedding dimension. math.AC/0702728.

3. Apel, J. (2003). On a conjecture of R. P. Stanley; part II—quotients modulo monomial ideals. Journal of Algebraic Combinatorics, 17, 57-74.

4. Björner, A., \& Wachs, M. (1997). Shellable nonpure complexes and posets. I. Transactions of American Mathematical Society, 349, 3945-3975.

5. Bruns, W., \& Herzog, J. (1995). On multigraded resolutions. Mathematical Proceedings of the Cambridge Philosophical Society, 118, 234-251.

6. Bruns, W., \& Herzog, J. (1996). Cohen-Macaulay rings, revised ed., Cambridge Univ. Press, Cambridge.

7. Conca, A., \& Herzog, J. (2003). Castelnuovo-Mumford regularity of products of ideals. Collecţia Matematică, 54, 137-152.

8. Dress, A. (1993). A new algebraic criterion for shellability. Beiträge zur Algebra und Geometrie, 34(1), 45-55.

9. Eagon, J., \& Reiner, V. (1998). Resolutions of Stanley-Reisner rings and Alexander duality. Journal of Pure and Applied Algebra, 130, 265-275.

10. Hachimori, M. Decompositions of two-dimensional simplicial complexes. Discrete Mathematics, in press.

11. Herzog, J., Hibi, T., \& Zheng, X. (2004). Monomial ideals whose powers have a linear resolution. Mathematica Scandinavica, 95(1), 23-32.

12. Herzog, J., Hibi, T., \& Zheng, X. (2004). Dirac's theorem on chordal graphs and Alexander duality. European Journal of Combinatorics, 25(7), 949-960.

13. Herzog, J., \& Popescu, D. (2006). Finite filtrations of modules and shellable multicomplexes. Manuscripta Mathematica, 121, 385-410.

14. Herzog, J., \& Takayama, Y. (2002). Resolutions by mapping cones. Homology, Homotopy and Applications, 4, 277-294. The Roos Festschrift, Vol. 2(2).

15. Jahan, A. S. (2007). Prime filtrations of monomial ideals and polarizations. Journal of Algebra, 312(2), 1011-1032.

16. Stanley, R. P. (1975). Cohen-Macaulay rings and constructible polytopes. Bulletin of the American Mathematical Society, 81, 133-135.

17. Stanley, R. P. (1982). Linear Diophantine equations and local cohomology. Inventiones Mathematicae, $68,175-193$.

18. Stanley, R. P. (1983). Combinatorics and commutative algebra. Birkhäuser, Basel.

19. Stanley, R. P. (2000). Positivity problems and conjectures in algebraic combinatorics. In V. Arnold, M. Atiyah, P. Lax, \& B. Mazur (Eds.), Mathematics: frontiers and perspectives (pp. 295-319). Providence: American Mathematical Society.

20. Terai, N. (2000). Generalization of Eagon-Reiner theorem and $h$-vectors of graded rings. Preprint. 